Journal of Applied Fluid Mechanics, Vol. 15, No. 2, pp. 551-561, 2022.

Available online at www.jafmonline.net, ISSN 1735-3572, EISSN 1735-3645.

https://doi.org/10.47176/jafm.15.02.33231

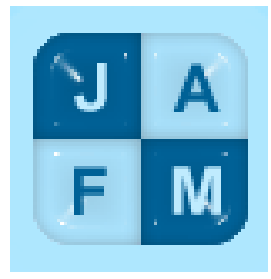

\title{
Improvement of Mass Transfer Rate Modeling for Prediction of Cavitating Flow
}

\author{
A. D. $\mathrm{Le}^{1 \dagger}$ and H. T. Tran ${ }^{2}$ \\ ${ }^{1}$ School of Aerospace Engineering, VNU-University of Engineering and Technology, Vietnam National \\ University (Hanoi), 144 Xuanthuy, Caugiay, Hanoi, Vietnam \\ ${ }^{2}$ Faculty of Aerospace Engineering, Le Quy Don Technical University, 236 Hoang Quoc Viet, Cau Giay,
} Hanoi, Vietnam

†Corresponding Author Email: anh.ld@vnu.edu.vn

(Received July 28, 2021; accepted October 21, 2021)

\begin{abstract}
This study proposes and investigates the impact of a modification, accounting for the influence of vortices and flow properties on the liquid rupture, to improve the modeling of mass transfer rate in cavitation. The threshold phase-change pressure is calculated by the fluid-saturated pressure at rest and the added vortex pressure term. The explicit simulation of the fully turbulent, homogeneous compressible, cavitating flow around the NACA0015 hydrofoil and the hemispherical body is performed. Saito cavitation model and Wilcox $k-\omega$ turbulence model are implemented for the evaluation of the proposed modification. The pressure coefficient distribution $-C_{p}$ and cavitation behavior, including the vapor formation-collapse processes and the flow mechanism, are investigated. The analysis shows that the present modification, coupled the local flow viscosity with the vorticity magnitude, making the cavitation model better sensitive to the flow condition. The modification has a weak impact on the steady sheet cavitation around a hemispherical body but is the key factor underlying the improvement in the predicted complex flow around the NACA0015 hydrofoil. In that, the predicted $-C_{p}$ and cavity structure around the hydrofoil is improved in comparison with the existing numerical data by other research groups and that by the Singhal turbulent pressure fluctuation model.
\end{abstract}

Keywords: Cavitation; Homogeneous model; NACA0015 hydrofoil; Saturated pressure; K- $\omega$ turbulent model; Vorticity magnitude; Hemispherical body.

\section{INTRODUCTION}

Cavitation is known as the generation and the condensation processes of vapor bubbles due to the local flow pressure drops below the saturated vapor pressure at a constant temperature. The vapor bubbles then collapse in the further downstream region, where the pressure recovers. Cavitation brings instability, noise, cavitation erosion, and the device's performance decreases. Literature has clarified the various type of cavitation, including bubble cavitation, sheet (attached) cavitation, sheetcloud cavitation, vortex cavitation, and supercavitation (See Brennen 1974). Typically, cavitation shows a strong link between the vapor-liquid interaction, the liquid quality, and the turbulence (Singhal et al. 2002).

Cavitation occurs in high Reynolds number flow, for which the flow is high unsteady and contains the shedding cavitating vortices phenomena with the periodic formation and collapse of the bubble clouds.
Previous studies showed that the existence of the vortex has a significant impact on the cavitation phenomena (Rambod et al. 1999; Bachmann et al. 2002; Arndt 2002; Li et al. 2012; Garam et al. 2020). These findings implied that the vortex core pressure is much smaller than the surrounding pressure, although the magnitude of the pressure drop is varied among these studies. Bachmann et al. (2002) calculated the vortex pressure drop of about $200 \mathrm{kPa}$ inside the vortex core with the radius of $0.25 \mathrm{~mm}$ using the PIV data and the inviscid vortex model. Similarly, Rambod et al. (1999) reported the pressure drop of $91 \mathrm{kPa}$ for the vortex core of $5 \mathrm{~mm}$. On contrary, Li et al. (2012) estimated a much smaller pressure drop of $3 \mathrm{kPa}$ inside the vortex core using the Rankine vortex model.

Regarding Computational Fluid Dynamics (CFD), the homogeneous model is a popular and effective approach for the numerical prediction of the cavitating flow (Iga et al. 2003; Coutier-Delgosha et al. 2003; Saito et al. 2007; Asnaghi et al. 2017, Anh 
et al. 2019a, b and 2021a, b; Zhou et al. 2019). For this approach, a transport equation for the vapor phase is implemented with the source term modeling the mass transfer rate between the phases. Several source term models had been proposed, including that based on the simplified Rayleigh-Plesset bubble dynamics equation (Schneer et al. 2001; Singhal et al. 2002; Zwart et al. 2004) and that based on the evaporation-condensation equation Saito et al. 2007; Ochiai 2011). In those source term models, the phase-change processes are mainly based on the pressure criteria while neglects the effect of the noncondensable gas, the liquid surface tension, the turbulence, and the vortices on the formation and the collapse of the cavitation bubbles. Although, several modifications were introduced to account for these effects on the simulation of cavitating flow such as the coupling of the Lagrange bubble model with the homogeneous model or the hybrid mixture model (Ghahramani et al. 2019, 2021), the original source term above is still widely used in the simulation community. That sometimes makes the unsatisfactory prediction of the complex cavitating flow with highly unsteady behavior (Kashiwada and Iga 2015; Ochiai 2011) and requires correction.

Recently, some attempts have been done to account for those effects. Singhal et al. (2002) accounted for the "turbulent pressure fluctuation" to the cavitation threshold phase-change pressure (CTPP) and applied it in the simulation of the cavitating flow for various objects. Although a satisfactory prediction with experimental data was produced for the attached cavitation, its acceptance is still limited for the unsteady cavitation and in the simulation community.

The shear stress pressure was numerically added to the CTPP for the prediction of the cavitating flow behavior in a nozzle (Martynov 2005; Som et al. 2010). For that flow type, the length scale is small and the velocity is very high, leading to the considerable shear velocity again the vortices. Similarly, Asnaghi et al. (2017) included the shear strain rate effect in the calculation of the CTPP. The additional pressure fluctuation, calculated by the strain rate and the fluid viscosity, was deployed to simulate the cavitating flow on the Delft Twist11 foil. Kashiwada and Iga (2015) coupled the laminar simulation with various pressure fluctuation models such as the Reynolds stress model, the Baroclinic torque model, the $\mathrm{Q}$ value model, and the strain rate model. As the report, the numerical $-C_{p}$ distribution on the NACA0015 hydrofoil slightly improved using the Reynolds stress model and the Q value model.

The studies above imply that the local flow behavior, either the turbulent viscosity or vortices, has an important impact on the phase-change processes and must be taken into consideration. Additionally, it is known that the simulation of cavitating flow mechanism is highly influenced by the numerical modeling of the turbulent viscosity. Bernard et al. (2006) demonstrated a link between the vortex structure and the flow mechanism near the wall. In that, the additional Reynolds stress is produced on the wall. Therefore, this would result in a higher cavitation criteria pressure but did not take into account in the previous studies (Martynov 2005; Som et al. 2010).

This study aims to account for the effect of viscosity and the vortices in the numerical simulation of cavitating flow. For which the cavitation threshold phase-change pressure (CTPP), a criterion for the cavitation to occurs, is calculated by the fluidsaturated pressure at rest and the added vortex pressure term. The cavitating flow around the NACA0015 hydrofoil (Saito et al. 2007) and the hemispherical body (Rouse and McNown 1948) are selected to evaluate the proposed modification. These flow fields, including both the attached and the unsteady sheet-cloud cavitation, are attractive test cases for the verification of the numerical method for the cavitation problem.

The density-based with explicit finite difference method (FDM) code is developed for the simulation of the full turbulence, homogeneous compressible flow. The Saito cavitation model (Saito et al. 2007), which is known to provide good numerical stability and produce sufficient results in the previous studies (Gnanaskandan and Mahesh 2016; Brandao et al. 2020; Anh et al. 2021a; Saito et al. 2007), and Wilcox $k$ - $\omega$ model (Wilcox 1994) are implemented. In addition, the result by the proposed modification is quantitatively compared with the numerical data by other research groups and by that obtained by the Singhal "turbulent fluctuation pressure" model. Finally, the empirical constant for the proposed modification is recommended.

\section{NUMERICAL METHOD}

\subsection{Homogeneous compressible liquid- vapor two-phase flow}

This study focuses on the fundamental understanding of the proposed modification threshold cavitation pressure, for which the time-averaged flow parameters such as the pressure and its coefficient are of interested justification. Thus, a two dimentional unsteady Reynolds-Averaged NavierStocks (2D URANS) simulation is performed because it can provide acceptable results with sufficient computational cost (Anh et al. 2021a,b; Chebli et al. 2021; Singhal et al. 2002; Asnaghi et al. 2017). The governing equations for the homogeneous compressible liquid-vapor two-phase flow are expressed as follows,

$$
\begin{aligned}
& \frac{\partial \rho}{\partial t}+\frac{\partial \rho u_{i}}{\partial x_{i}}=0, \\
& \frac{\partial \rho u_{i}}{\partial t}+\frac{\partial\left(\rho u_{i} u_{j}+\delta_{i j} p\right)}{\partial x_{j}}=\frac{\partial \tau_{i j}}{\partial x_{i}}, \\
& \frac{\partial \rho Y}{\partial t}+\frac{\partial \rho u_{i} Y}{\partial x_{i}}=\dot{m} .
\end{aligned}
$$

Here, $\rho, p$, and $u_{i}$ are the density, the pressure, and the velocity components of the mixture, respectively. In addition, $Y$ is the vapor mass fraction and $\dot{m}$ is the cavitation net mass transfer rate. The $\delta_{i j}$ is the Dirac function. The viscous shear stress $\tau_{i j}$ is computed by, 
A. D. Le and H. T. Tran / JAFM, Vol. 15, No. 2, pp. 551-561, 2022.

$$
\tau_{i j}=\left(\mu+\mu_{t}\right)\left(\frac{\partial u_{j}}{\partial x_{i}}+\frac{\partial u_{i}}{\partial x_{j}}-\frac{2 \delta_{i j}}{3} \frac{\partial u_{k}}{\partial x_{k}}\right)
$$

With the molecule viscosity of mixture $\mu$ is calculated as follows (Anh et al. 2021a,b; Gnanaskandan and Mahesh 2016; Brandao et al. 2020),

$\mu=(1-\alpha)(1+2.5 \alpha) \mu_{l}+(1-\alpha) \mu_{v}$.

In this equation, $\alpha$ is the vapor void fraction. The subscripts $v$ and $l$ denote the vapor phase and liquid phase, respectively. The turbulence term is denoted by the subscript $t$. Both the liquid and the vapor phases assume compressible fluid. The liquid density $\rho_{l}$ and the vapor density $\rho_{v}$ are calculated using the Tamman equation (Anh et al. 2019a, Iga et al. 2003) and the ideal gas equation, respectively.

Following relation between the vapor void fraction $\alpha$ and the vapor mass fraction $Y$ is obtained,

$\rho Y=\rho_{v} \alpha$,

$\rho(1-Y)=\rho_{l}(1-\alpha)$.

The equation of state then expresses by,

$p=\frac{(1-Y) \rho K_{l} p\left(T_{0}+T_{c}\right)}{p+p_{c}}+\rho R_{v} Y T_{0}$.

Here, $p_{c}=2231.2 \mathrm{MPa}, T_{c}=3348.9 \mathrm{~K}$, and $K_{l}=614.45 \mathrm{Jkg}^{-1} \mathrm{~K}^{-1}$ in present study. The vapor constant is $R_{v}=461 \mathrm{~J} / \mathrm{kg}^{-1} \mathrm{~K}^{-1}$. Besides, $T_{0}$ is the freestream temperature of the water.

The sound speed of mixture $C$ is an important parameter in a two-phase flow simulation. The present numerical speed of sound agreed numerically with Karplus's measured data for water at room temperature (See Iga et al. 2003).

\subsection{Turbulent modeling}

Wilcox's two-equation turbulence model (Wilcox 1994), expressed for the turbulent kinetic energy $k$ and the turbulent specific dissipation rate $\omega$, is coupled with the governing equations Eq. (1). This model has a good performance in the flow with the adverse pressure gradient and is successfully applied in our previous studies (Anh et al. 2019a, b; 2021a, b). The modification of the turbulent viscosity $\mu_{t}$ is adopted as follows (Coutier-Delgosha et al. 2003; Chebli et al. 2021),

$$
\begin{aligned}
& \mu_{t}=f \frac{\rho k}{\omega}, \\
& f=\frac{\rho_{v}+(1-\alpha)^{n_{T}}\left(\rho_{l}-\rho_{v}\right)}{\rho_{v}+(1-\alpha)\left(\rho_{l}-\rho_{v}\right)} .
\end{aligned}
$$

In this study, the effect of turbulent viscosity on the simulation of the cavitating flow is approached through the implementation of the correction parameter $n_{T}$.

\subsection{Saito mass transfer model}

Saito cavitation model (Saito et al. 2007) is employed to calculate the net mass transfer rate $\dot{m}$ in cavitation as follow,

$$
\dot{m}=\left\{\begin{array}{l}
\frac{C_{e c}[\alpha(1-\alpha)]^{2} \max \left(0, p_{v}{ }^{*}-p\right)}{\sqrt{2 \pi R_{v} T_{0}}} \frac{\rho_{l}}{\rho_{v}} \\
\frac{C_{e c}[\alpha(1-\alpha)]^{2} \min \left(0, p_{v}{ }^{*}-p\right)}{\sqrt{2 \pi R_{v} T_{0}}}
\end{array} .\right.
$$

In this model, $C_{e c}=0.1$, suggested by Saito et al. (2007), is the reference phase-change empirical parameter. In addition, $p_{v}{ }^{*}$ is the CTPP and is discussed in detail in subsection 2.4.

\subsection{Modification of cavitation threshold phase-change pressure}

Cavitation usually occurs in high Reynolds number flow. In most cases, the flow is high unsteady and contains a shedding cavitating vortices phenomena with the periodic formation and the collapse of the bubble clouds. Firstly, the attached vapor bubble generates in the low-pressure region near the object. When the cavitation develops, the reentrance flow occurs that breaks the main cavity and creates cloud cavitation. In that, the flow is highly recirculated and is influenced by the vortex strength $\Gamma$ (Pelz et al. 2017). Arndt (2002) modified a cavitation inception index $\sigma_{i}=\left(p_{\infty}-p_{v}\right) /\left(0.5 \rho U^{2}\right)$ by accounting for the strength of vortex $\Gamma$ using the Rankine vortex model as follow,

$\sigma_{i}-\left[\frac{\Gamma}{2 \pi r U}\right]^{2}=-C_{p \min }$.

Here, $-C_{p \min }$ is the minimum pressure coefficient at the vortex core with radius $r$ and $U$ is the free-stream velocity. The right-hand side of Eq. (8) can be rewritten as follows,

$$
\frac{p_{\infty}-\left(p_{v}+\frac{1}{2} \rho\left[\frac{\Gamma}{2 \pi r}\right]^{2}\right)}{\frac{1}{2} \rho U^{2}}=\frac{p_{\infty}-\left(p_{v}+p_{a d d}\right)}{\frac{1}{2} \rho U^{2}} .
$$

In Eq. (9), term $0.5 \rho[\Gamma / 2 \pi r]^{2}$ has the pressure dimension and is the additional pressure padd caused by the vortices. The term $\left(p_{v}+p_{a d d}\right)$ responds to the CTPP $p_{v}{ }^{*}$ in Eq. (7).

Besides, the vortex strength $\Gamma$ can calculate using the Rankine vortex model as follows,

$\Gamma=2 \pi r U$.

The additional vortex pressure, $p_{a d d}$, is then obtained by substituting $\Gamma$ in Eq. (10) as follow,

$$
p_{\text {add }}=\rho U^{2} \square c_{1} \rho k \text {. }
$$

The introduction section implies a high interaction between the cavitation mechanism, vortex, and turbulence. Asnaghi et al. (2017) mentioned that "In flowing fluids, the viscous stresses appear in the stress tensor, and therefore they will affect the magnitude and direction of the forces acting on the liquid pocket". The maximum eigenvalue of the stress tensor, summing of the static pressure tensile and viscous stress, then considered as the criteria of phase-change in cavitation as in Eq. (12), 


$$
p_{v}^{*}=p_{v}+\mu \dot{\gamma}
$$

In this equation, $\gamma$ is the shear strain rate. Singhal et al. (2002) calculated the CTPP $p_{v}{ }^{*}$ using the saturated vapor pressure at rest $p_{v}$ and the "turbulent pressure fluctuation" $p_{\text {tur }}^{\prime}=0.195 \rho k$ as follows,

$$
p_{v}^{*}=p_{v}+0.195 \rho k
$$

The padd in Eq. (11) is almost identical to the "turbulent pressure fluctuation" $p_{\text {tur }}$ in Eq. (13) which has a similar form of Johnson-King's shear stress formula $\tau=a_{1} \rho k$ (Menter, 1992), an indirect way to express the viscous stress $\mu \gamma$ in Eq. (12).

Bernard et al. (2006) showed a link between the vortex structure and Reynolds shear stress near the wall region. The vortex provides the additional Reynolds stress on the wall where cavitation usually occurs. Therefore, that could significantly influence cavitation behavior. Based on the works of Asnaghi et al. (2017), using the same methodology as in the turbulent modeling (Menter 1992) with focusing on the vortex effect, the cavitation threshold pressure with additional vortex pressure $p_{\text {add }}$ is expressed by replacing the shear strain rate $\dot{\gamma}$ by the vorticity magnitude $|\Omega|$ as follow for the 2D flow,

$$
p_{v}^{*}=p_{v}+C_{v} \mu_{e f f}\left|\frac{\partial v}{\partial x}-\frac{\partial u}{\partial y}\right| .
$$

In this equation, the $p_{a d d}$ is important if either the effective viscosity $\mu_{\text {eff }}$ or the vorticity magnitude is large. For instant, the shedding cloud cavitation around the NACA0015 hydrofoil in this study is the case of the high effect of the vortices. In addition, $C_{v}$ is the empirical constant, and its sensitivity to the numerical results is investigated in detail hereafter.

\subsection{Solution procedure}

The numerical simulation code, based on the finite difference method, was written in FORTRAN language. The density-based algorithm with the explicit Harten-Yee second-order upwind Total Variation Diminishing (TVD) scheme is employed (Yee 1987). This scheme ensures numerical stability for the high compressibility flow with the large discontinuity of flow parameters in cavitation. The second-order space-centered scheme is used to discretize the viscous terms. Strang's splitting step (Yee 1987) is applied to time discretization to obtain the second-order accuracy. For the wall region, Allmaras's wall function is deployed (Allmaras et al. 2012). The detail of the numerical method, validated successfully for cavitation flow around various objects, can refer to our previous works (Anh et al. 2021a, b).

\section{RESULTS AND DISCUSSION}

In this section, the proposed modification for the CTPP $p_{v}{ }^{*}$ in Eq. (14), named as "vortex pres. model" hereafter, is evaluated. The experimental study of cavitating flow around a NACA0015 hydrofoil (Saito et al. 2007) and a hemispherical body (Rouse and McNown 1948) are selected for the numerical benchmarking. In subsection 3.1, the computational configuration, the grid detail, and the boundary conditions are illustrated. In subsection 3.2, the simulation of unsteady cavitating flow around the hydrofoil is performed at different flow cavitation numbers $\sigma=1.8,1.4$, and 1.0. This work aims to evaluate the impact of the proposed modification, the sensitivity of parameter $C_{v}$, and turbulent correction parameter $n_{T}$ on the predicted flow field. Finally, the proposed modification model is validated for the attached cavitation flow around the hemispherical body in subsection 3.3.

\subsection{Computational configuration and boundary condition}

The configuration of the computational domains for the NACA0015 hydrofoil and the hemispherical body is illustrated in Fig. 1. For NACA0015 hydrofoil, the attack angle $A o A$ is $8 \mathrm{deg}$. The hydrofoil with the chord length $c=0.15 \mathrm{~m}$ locates at $4 c$ from the inlet boundary and $1 c$ from two outer boundaries. The outlet boundary is at $10 c$ from the inlet. For the second object, the hemispherical body has a diameter $D=0.025 \mathrm{~m}$. The computational domain has $10 D$ in height and $30 D$ in length. Notably, the simulation is performed for half of the hemispherical body because of the axisymmetric assumption.

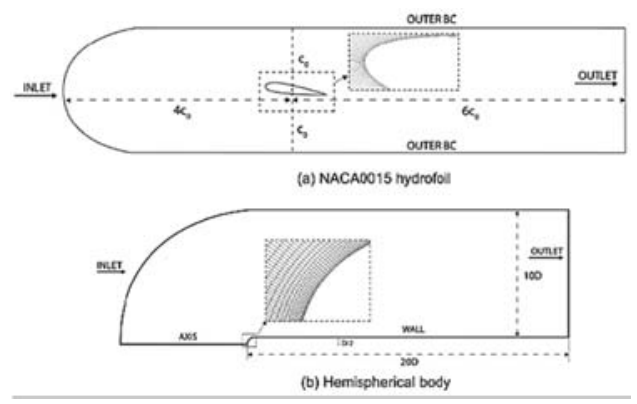

Fig. 1. Configuration of computational domain: (a) NACA0015 hydrofoil and (b) Hemispherical body.

For both objects, the uniform velocity corresponding to Reynolds flow number Re is specified at the inlet boundary. In addition, the initial void fraction $\alpha_{0}=$ 0.01 suggested by Iga et al. (2003) and turbulent intensity $I=2 \%$ (Ducoin et al. 2012) are implemented. The non-slip and Newmann boundary condition is applied to the object's surfaces. The constant pressure, calculated from the flow cavitation number $\sigma$, is applied to the outlet boundary. Besides, for NACA hydrofoil, the slip condition is used for the outer boundaries. The water temperature assumes $T_{0}=298 \mathrm{~K}$. The detail of the numerical conditions is shown in Table 1.

An orthogonal boundary fitted grid is generated for two computation domains. To archive the averaged $y^{+}$of over 30 , the grid is clustered to the hydrofoil surface and hemispherical body with distance $\Delta s=1$ $\mathrm{x} 10^{-4} \mathrm{~m}$ and $\Delta s=8 \times 10^{-5} \mathrm{~m}$, respectively. At first, 
the grid convergence study is performed. As the result, the grid of $309 \times 85$ points and $279 \times 85$ points are used for the NACA0015 hydrofoil (141 grid points on hydrofoil) and the hemispherical body ( 80 points on the headform), respectively. The numerical time step-size is set to $2 \times 10^{-7} \mathrm{~s}$, corresponding to CFL $<0.5$ for the hydrofoil and CFL $<0.8$ for the hemispherical body simulations, respectively.

Table 1 Computational conditions for NACA0015 hydrofoil and hemispherical body.

\begin{tabular}{|c|c|c|}
\hline $\begin{array}{c}\text { Attack angle } \\
{[\mathrm{deg}]}\end{array}$ & $\begin{array}{c}\text { Reynolds } \\
\text { number }\end{array}$ & $\begin{array}{c}\text { Cavitation } \\
\text { number }\end{array}$ \\
\hline \multicolumn{3}{|c|}{ NACA0015 hydrofoil } \\
\hline 8 & $1.3 \times 10^{6}$ & $1.0,1.4,1.8,3.0$ \\
\hline \multicolumn{3}{|c|}{ Hemispherical body } \\
\hline 0 & $1.36 \times 10^{5}$ & $0.2,0.4,0.5,0.8$ \\
\hline
\end{tabular}

For instance, Fig. 2 shows the comparison of pressure coefficient distribution $-C_{p}=2\left(p-p_{\infty}\right) /\left(\rho U^{2}\right)$ on the hydrofoil surface between three grids: grid 1 ( $221 \times 70$ points), grid 2 ( $309 \times 85$ points), and grid 3 (410 x 100 points). The non-cavitating flow condition at $\sigma=3.0$ is considered for the numerical benchmarking. The simulation is performed using the "Singhal pres. model" in Eq. (13) to calculate the CTPP $p_{v}{ }^{*}$. As the result, a similar $-C_{p}$ tendency produces between the three grids that are in good agreement with experimental data (Ochiai et al. 2011). It implies the acceptance of the present numerical method for capturing the flow field around a hydrofoil. In addition, the predicted pressure coefficient $-C_{p}$ is almost identical between grid 2 and grid 3. Therefore, grid 2 is sufficient to use in further simulations in this study.

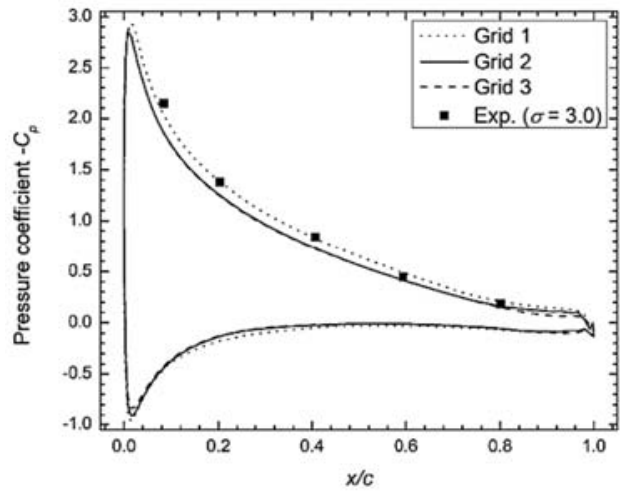

Fig. 2. Grid sensitive study: comparison of pressure coefficient distribution $-C_{p}$ on NACA0015 hydrofoil between three grids and measured data (Ochiai et al. 2011).

\subsection{Cavitating flow around NACA0015}

In this subsection, the cavitating flow around the NACA0015 hydrofoil is simulated. Different flow cavitation numbers $\sigma=1.8,1.4$, and 1 , corresponding to different cavitating flow types, are performed. The numerical averaged pressure coefficient $-C_{p}$, taken in the 5 cycles of the cavity break-off after the effect of the initial condition come-off, is compared quantitatively with experimental data (Ochiai et al. 2011). The purpose of this works is to clarify the impact of the proposed modification and the sensitivity of model parameter $C_{v}$ on the flow mechanism.

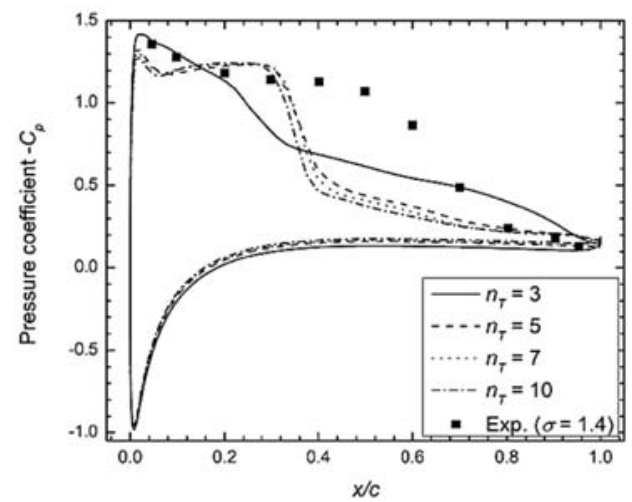

Fig. 3. Influence of turbulent viscosity: the effect of correction parameter $n_{T}$ (exp. data - Ochai et al. 2011).

It is known that the simulation of cavitation is highly affected by the numerical modeling of the turbulent viscosity. Figure 3 depicts the $-C_{p}$ distribution on the hydrofoil with different $n_{T}$ values ranged from $n_{T}=3$ to $n_{T}=10$ at the flow condition $\sigma=1.4$. The simulations are performed using Singhal pres. model. Regarding the leading edge region $x / c<0.2$, the $-C_{p}$ is in good agreement with the measured data for $n_{T}=3$ while it is underestimated for $n_{T}$ at a higher value. Between $0.2<x / c<0.4$, the region with high $-C_{p}$ increases for $n_{T}$ of greater than 3 and better matches the measured data at $x / c=0.3$ before dramatically decreasing to the reference value. Here, the reference value denotes the $-C_{p}$ calculated by the reference pressure at the outlet boundary. The overall profile is far different from the measured data. In addition, high $n_{T}$ results in a faster decrease of $-C_{p}$. This causes by that the turbulent viscosity decreases when the $n_{T}$ increases. The reentrance flow is stronger and the cloud cavity is faster detached away from the hydrofoil surface, resulting in the rapid increase of the local pressure on the hydrofoil at this region. The predicted $-C_{p}$ improves slightly in the mid-chord of hydrofoil $(0.4<x / c<0.7)$ for $n_{T}=3$. In addition, the grid resolution effect was performed for Grid 2 and Grid 3 above. As the result, the grid resolution has some effects the pressure distribution. Although there is a slight discrepancy of $-C_{p}$ distribution between the two grids at the mid-chord region $(0.3<x / c<0.6)$, the $-C_{p}$ tendency is almost similar between the two grids. Besides, Ochiai et al. (2011) intensively conducted the grid resolution effect on the prediction of $-C_{p}$ of the same problem. The cavity did get longer as the grid resolution 


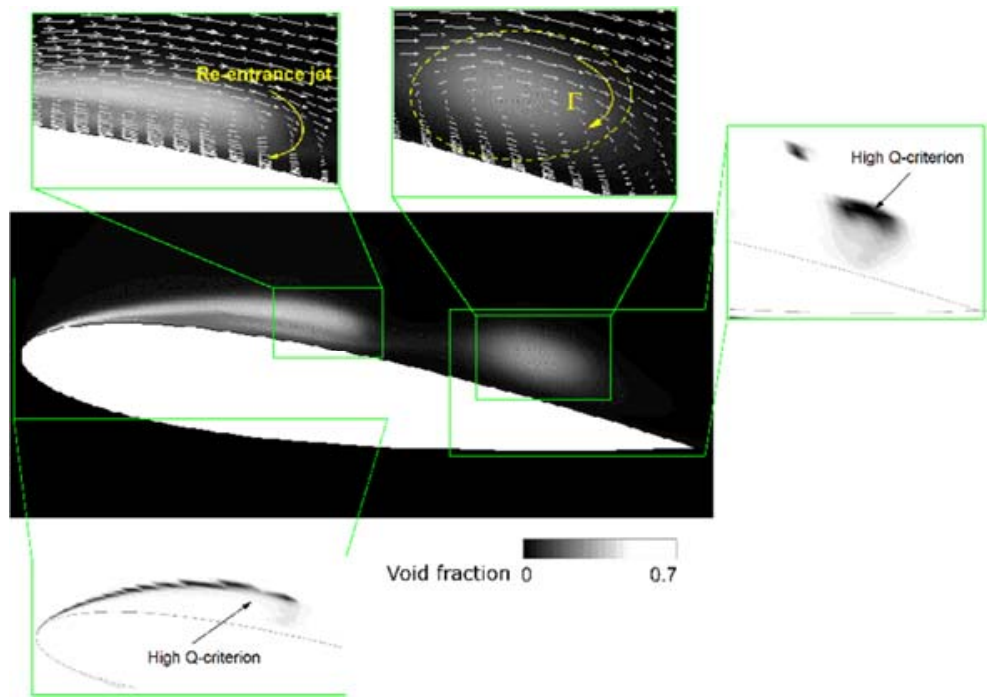

Fig. 4. Instantaneous numerical vapor void fraction, velocity vector, and Q-criterion (from 0 to 1 ) on NACA0015 hydrofoil for $\sigma=1.4$.

increased. However, similar to this study, the almost similar tendency of $-C_{p}$, which underestimated the experimental data, was still reproduced even at a very fine grid. The above results indicate an important effect of turbulent viscosity on the numerical prediction of cavitating flow. In addition, with the satisfactory prediction of the leading-edge pressure and the improvement of mid-chord pressure, the correction parameter $n_{T}=3$ is considered for simulating the cavitating flow on a hydrofoil in this study. This parameter was also recommended in previous studies by Ducoin et al. (2012) and Anh et al. (2019a).

Figure 4 shows the instantaneous numerical vapor void fraction and velocity vector on NACA0015 hydrofoil for flow cavitation number $\sigma=1.4$. The result is obtained by present vortex pres. model with the empirical constant $C_{v}=10$ as a reference value. The attached vapor occurs near the hydrofoil leading edge and extends to the mid-chord region. The cavity is detached away from the hydrofoil surface due to the reentrance flow. The reentrance jet breaks the main cavity and releases the small cloud cavitation with the recirculation flow. In addition, the normalized Q-criterion is high nearby the cloud cavity region and at the main cavity surface, where the closed circulation flow is observed. Notably, the plotted normalized Q-criterion is from 0 to 1 . Although the Q-criterion is mainly designed to identify the $3 \mathrm{D}$ vortex structure, the visualization of the Q-criterion in the present 2D study supposes the existence of vortices in the current flow on the hydrofoil.

Figure 5 depicts the comparison of the numerical pressure coefficient distribution $-C_{p}$ between present vortex pres. model and measured data for the flow cavitation numbers $\sigma=1.4$. In addition, the reference numerical data by other research groups (Ochiai 2011; Kashiwada and Iga 2015) and by the present numerical method using Singhal pres. model are quantitatively compared. The distribution of $-C_{p}$ is

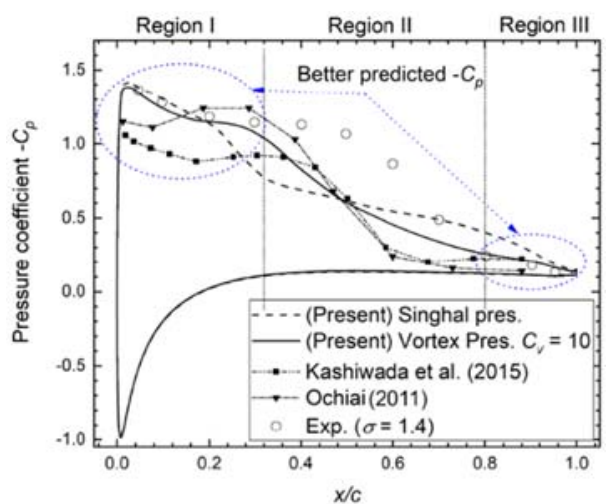

Fig. 5. Comparison of $-C_{p}$ on hydrofoil for $\sigma=1.4$ between the vortex pres. model, the Singhal pres. model, the experimental data (Ochiai et al. 2011), and the existing reference numerical data.

divided into three regions: (I) the leading edge region $x / c<0.3$, (II) the mid-chord region $0.3<x / c<0.8$, and (III) the trailing edge region $0.8<x / c$. For the trailing edge region (III), a good prediction with experimental data is produced for all numerical models. The difference becomes significant in two other regions. At the leading edge region (I), the $-C_{p}$ is well captured by the present numerical method with both Singhal pres. model and vortex pres. model. An improvement in $-C_{p}$ is produced by the vortex pres. model at $x / c \sim 0.3$. At the same time, the larger underestimation of $-C_{p}$ is produced in the references numerical data. For the mid-chord region (II), the discrepancy between the measured data and all numerical data becomes large. All models fail to capture the measured $-C_{p}$ profile correctly. The $-C_{p}$ decreases rapidly to around the reference value at $x / c>0.4$ in references numerical data that is a far difference from the experiment. This behavior is similar to the case of $n_{T}>3$ in Fig. 3. In contrast, the $-C_{p}$ decreases slower in the present vortex pres. model and the Singhal pres. model. For which the 
A. D. Le and H. T. Tran / JAFM, Vol. 15, No. 2, pp. 551-561, 2022.
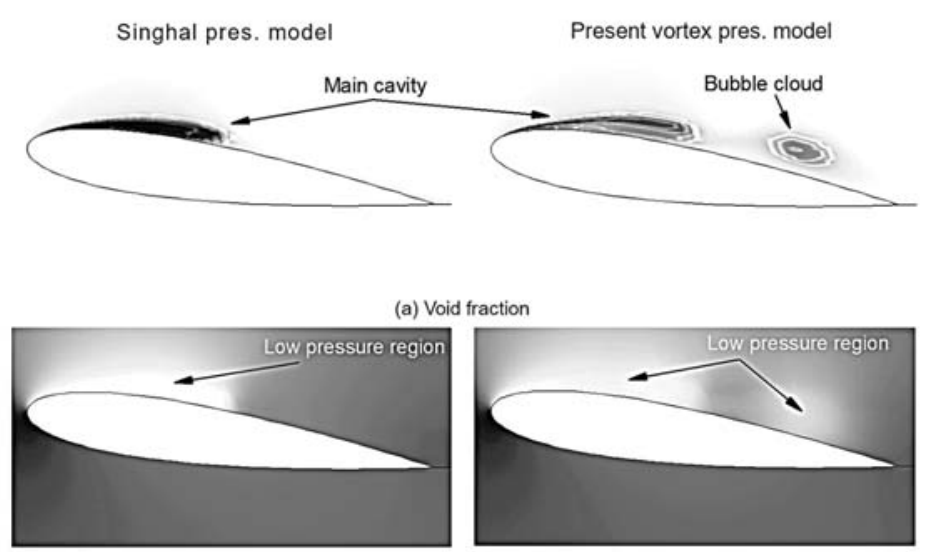

(b) Local pressure

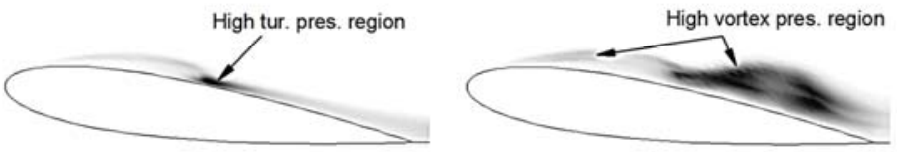

(c) Additional pressure
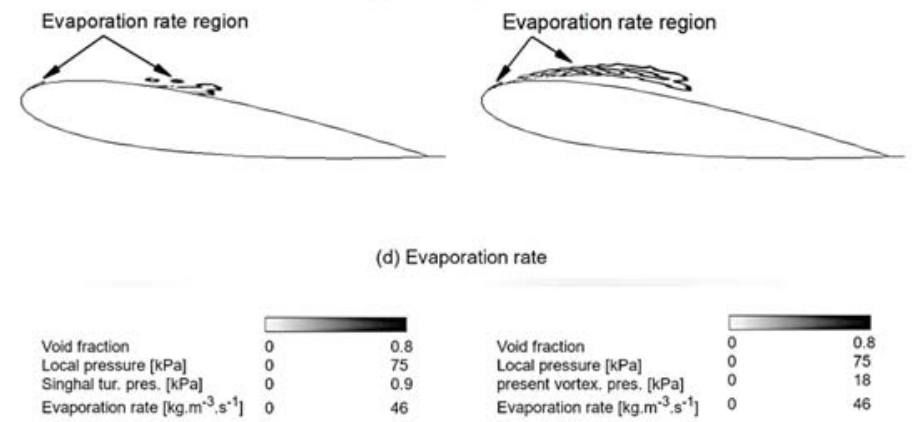

Fig. 6. Instantaneous contour of the flow parameters on the hydrofoil by the Singhal pres. model and the vortex pres. model for $\sigma=1.4$.

$-C_{p}$ profile shows an improvement compared with the references numerical data in the mid-chord region (II) with $x / c>0.5$. Notably, an almost identical $-C_{p}$ profile is produced by the vortex pres. model and the Singhal pres. model near the hydrofoil leading edge region (I). In addition, the vortex effect supposes small in the leading edge region and the modeling of turbulent viscosity is the flow physics underlying the better predicted $-C_{p}$ in the region (I) by the present simulation. Moreover, the laminar simulation was performed in the work of Kashiwada et al. (2015), which produced the large cloud cavitation in the mid-chord region than in the present simulation. Thus, that results in a better agreement with measured data in their simulation at $0.4<x / c<$ 0.5 comparisons to that in present data.

More detail can be seen in Fig. 6, which illustrates the instantaneous contours of the void fraction, the local pressure, the additional pressure term, the evaporation rate, and the condensation rate on hydrofoil by the Singhal pres. model and the vortex pres. model at $\sigma=1.4$. The additional pressure term indicates the turbulent pressure fluctuation $p^{\prime}$ tur in Eq. (13) and the vortex pressure $p_{\text {add }}$ in Eq. (14). The sheet cavitation attaches to the hydrofoil surface from the leading edge to around $0.5 c$ in Singhal pres. model. With an identical cavity length, the additional cloud cavitation is produced in the vortex pres. model.

The higher $-C_{p}$ then results on the hydrofoil surface compared to that in the Singhal pres. model. In addition, the higher additional pressure term is produced around the cloud cavity and attached cavity surface in the vortex pres. model. The CTPP $p_{v}{ }^{*}$ is thus higher, resulting in a higher evaporation rate for the vortex pres. model. In which, the water is evaporated at the hydrofoil leading edge and along the attached cavity surface. In contrast, the evaporation rate occurs only at the hydrofoil leading edge and behind the cavity trailing edge for the Singhal pres. model.

Figure 7 illustrates the contours of the vapor void fraction (a), the vortex pressure contour with streamline (b), and the effective viscosity (c) around the hydrofoil at different flow times at $\sigma=1.4$ by the vortex pres. model. The cavitation structure snapshot (d) is included for the qualitative comparison. The vapor bubble firstly appears at the hydrofoil leading edge. As the attached cavitation (AC) grows, the 
(a) Void fraction
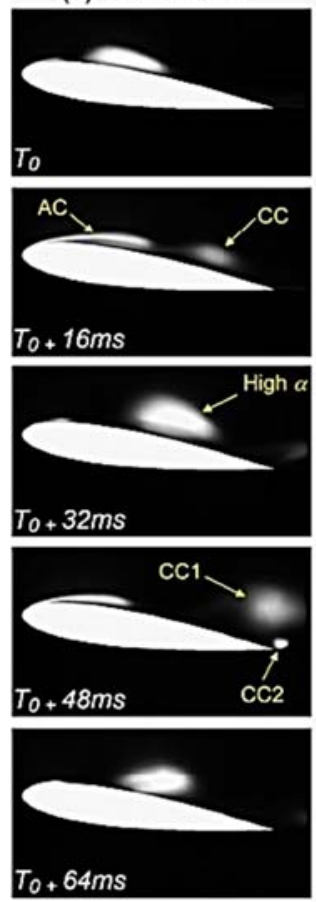

(b) Vortex pres.
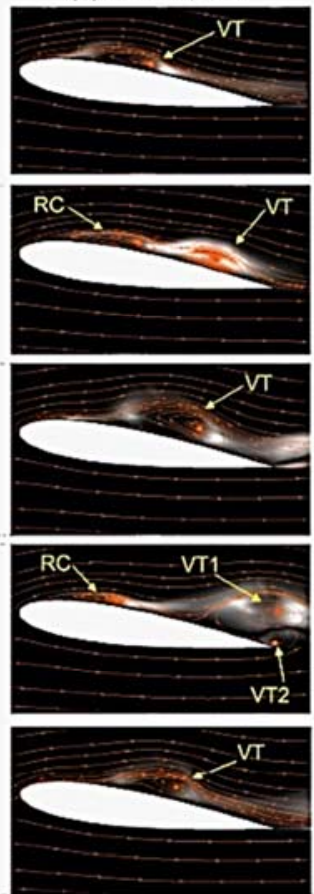

(c) Effective vis.
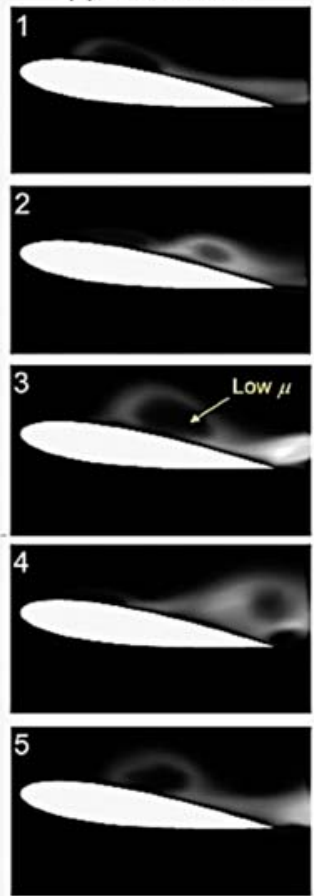

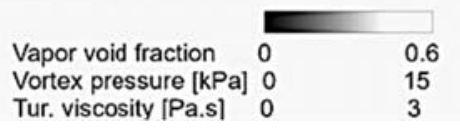

(d) Experimental snapshot
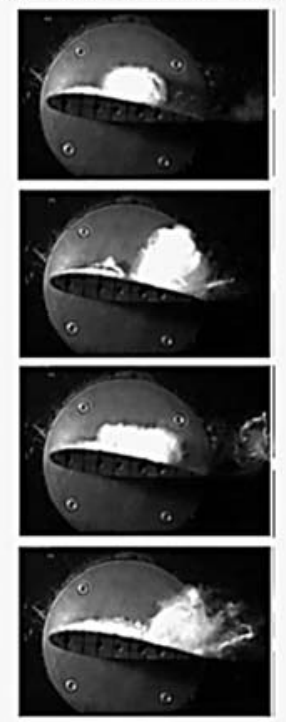

Fig. 7. Contour of the vapor void fraction (a), the vortex pressure contour with streamline (b), the effective viscosity (c), and the experimental snapshot (Ochiai et al. 2011) around the hydrofoil at different flow times for $\sigma=1.4$.

recirculation $(\mathrm{RC})$ and the reentrance jet occur. The reentrance flow breaks the attached cavity and releases the cloud cavitation bubble (CC) in Fig. 7(a). The vortex (VT) with a high recirculation flow presents inside the cloud cavity. The vortex pressure $p_{\text {add }}$ is high around the cloud cavitation, as shown in Fig. 7(b). The smaller value produces inside the cloud cavity, for which the high void fraction and the low mixture effective viscosity are observed as in Figs. 7(a and $\mathrm{c})$. The maximum vortex pressure $p_{\text {add }}$ of $18 \mathrm{kPa}$ is added to the CTPP $p_{v}{ }^{*}$, which has the same order as in the experiment (Rambod et al. 1999; Bachmann et al. 2002; Li et al. 2012). When the cloud cavitation (CC1) moves to downstream, the small cloud cavitation (CC2) is generated at the hydrofoil trailing edge as in Fig. 7(a-4). This is caused by the secondary vortex (VT2) appears at the trailing edge due to the flow interaction between the large cloud vortex (VT1) and the flow from the pressure side. The numerical cavity structure agrees qualitatively with the experimental snapshot, however, offsets to a smaller cloud cavity area due to the difference in the time-averaged $-C_{p}$ in the region (II), as shown in Fig.5.

In the simulations above, the $C_{v}=10$ is used as the reference empirical constant for the vortex pres. model in Eq. (14). Since the $C_{v}$ can change the magnitude of the CTPP $p_{v}{ }^{*}$, the cavitation mechanism is thus significantly influenced. In this subsequence, the effect of the empirical constant $C_{v}$ is investigated. Notably, increasing the $C_{v}$ to the value higher than 100 does not change the result significantly in the present study. Thus, the data is plotted here for $C_{v}$ from 1 to 100. Figure 8(a) illustrates the $-C_{p}$ profile on hydrofoil with different $C_{v}$ at $\sigma=1.4$. Regarding $C_{v}=1$, the predicted $-C_{p}$ profile is almost identical to the result obtained by the Singhal pres. model, as shown in Fig. 5. When $C_{v}$ increases, the region of high $-C_{p}$ expands further downstream but the $-C_{p}$ decreases significantly at the hydrofoil leading edge region (I). The $-C_{p}$ profile is nearly identical between $C_{v}=50$ and $C_{v}=100$. More detail can be seen in Fig. 8(b), showing a periodic formation and collapse of the vapor cavitation on hydrofoil with $C_{v}=50$. The sheet cavity almost attaches to the hydrofoil surface instead of the detached cavity with $C_{v}=10$, as shown in Fig. 4, resulting in a better predicted $-C_{p}$ near $x / c=0.4$. Opposed to $C_{v}=10$, during a cycle, the sheet cavitation appears without the cloud cavitation. Hence, the $-C_{p}$ is underestimated in the rest of the hydrofoil. It is evident that the numerical results by the vortex pres. model better agrees with experimental data with $C_{v}=10$.

Figure 9 depicts the $-C_{p}$ distribution on the hydrofoil surface at the cavitation number $\sigma=1.8$ (a) and $\sigma=1.0$ (b). Regarding $\sigma=1.8$ (a), the attached cavitation is produced. The $p_{\text {add }}$ results in a high 


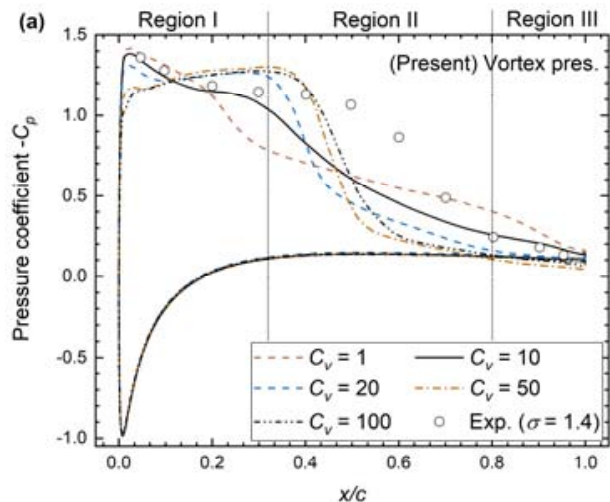

(b)
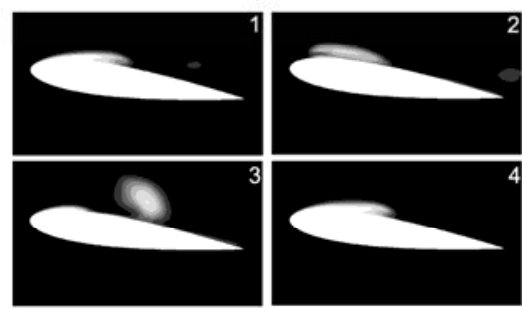

Fig. 8. (a) Influence of empirical constant $C_{v}$ on $C_{p}$ (Exp. data - Ochiai et al. 2011) and (b)

instantaneous vapor void fraction contour on hydrofoil for $C_{v}=\mathbf{5 0}$ (the order of image is from 1 to 4 with interval time $\Delta t=8 \mathrm{~ms}$ ).
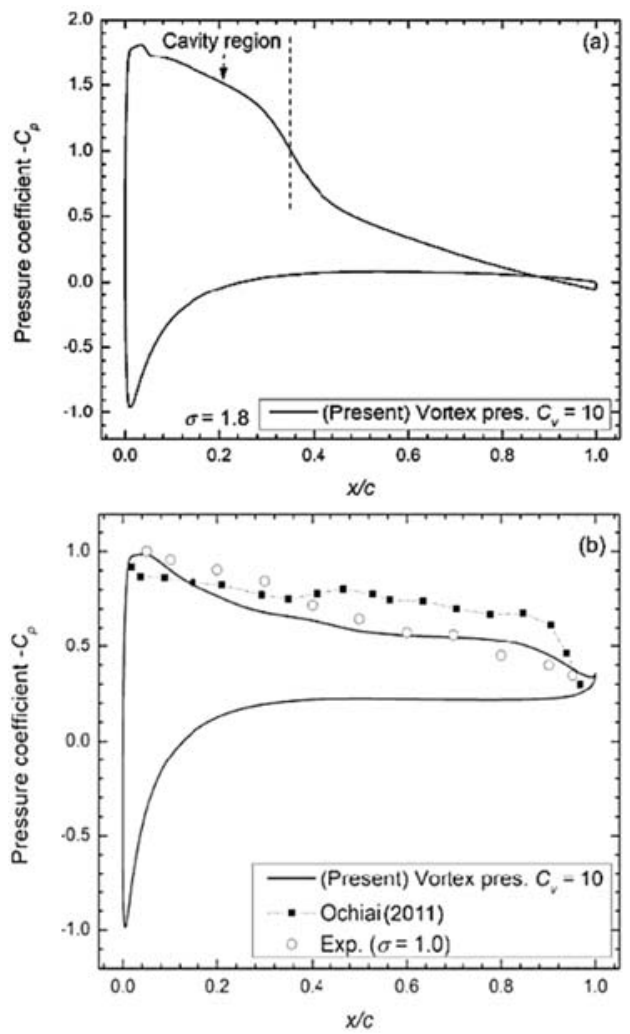

Fig. 9. Pressure coefficient $-C_{p}$ distribution on the hydrofoil at (a) $\sigma=1.8$ and (a) $\sigma=1.0$ (Exp. data - Ochiai et al. 2011).
CTPP $p_{v}{ }^{*}$ around the attached cavity surface that decreases the $-C_{p}$ inside the cavity region, as shown in Fig. 9(a). For $\sigma=1.0$ (b), the sheet-cloud cavitation is produced with the large cloud cavitation around the hydrofoil. Similar to $\sigma=1.4$, the padd is high around the cloud cavitation region, where the flow recirculates. The predicted $-C_{p}$ agrees with the experimental data and shows a slight improvement compared with the Ochiai numerical data (Ochiai 2011). The results above suggest that the correction parameter $C_{v}=10$ is suitable for the numerical simulation of the cavitating flow around the hydrofoil in this study.

\subsection{Cavitating flow around Hemisperical body}

In this subsection, the proposed modification with the empirical constant $C_{v}=10$ is applied to predict the cavitating flow around the hemispherical body. The numerical result is quantitatively compared with the experimental data by Rouse and McNown (1948). Figure 10 depicts the comparison of the pressure coefficient $C_{p}$ between the present simulation and the measured data at different cavitation numbers $\sigma$ ranged from 0.2 to 0.8 . According to the result, the steady cavitation results in all simulations. An acceptable agreement with experimental data is produced in both tendency and value. The peak pressure captures reasonably at $\sigma=$ 0.4 and 0.2 . Notably, an almost identical $C_{p}$ profile is produced between the present numerical data using the vortex pres. model and the Singhal pres. model for the present object, indicating that the proposed modification has a weak impact on the steady sheet cavitation, where the effect of vorticity is small.

\section{CONCLUSION}

In this paper, the numerical simulation of cavitating flow around the NACA0015 hydrofoil and the hemispherical body is performed. A modification to improve the modeling of the cavitation mass transfer rate is proposed and is evaluated.

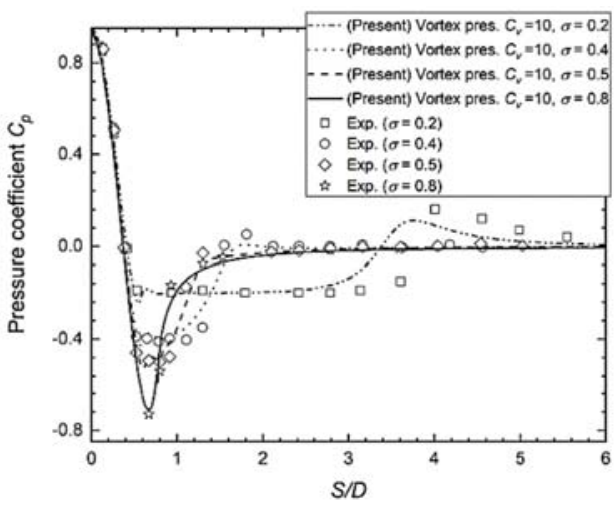

Fig. 10. Comparison of pressure coefficient distribution on the axisymmetric body in numerical prediction and experiment (Rouse and McNown 1948) for different cavitation number. 
According to the results, the present modification, coupled the local flow viscosity with the vorticity magnitude, making the cavitation model better sensitive to the flow condition. In that, an improvement and acceptable agreement with measured data is produced for the benchmarking cases in this study. The model empirical constant $C_{v}=10$ is recommended for the present modification model.

The present modification has a weak impact on the flow field with low vortices. In that, an almost identical result is produced between the present modification and the popular Singhal pres. model.

On contrary, the proposed modification shows effectiveness to predict the unsteady cavitating flow and is the key factor underlying the improvement in the prediction of flow parameters around the hydrofoil. The criteria cavitation threshold pressure and the evaporation rate increase around the cavity surface, resulting in a better pressure coefficient profile at the leading edge regions in comparison with the existing numerical data by other research groups.

Since the proposed modification is currently tested only for the Saito cavitation model, it expects that the present modification is useable for different cavitation models and flow types. In addition, the predicted pressure coefficient is still underestimated in the mid-chord region in NACA0015 hydrofoil simulation, suggesting the remains room for further improvement of the mass transfer model. This can be done by accounting for both shear strain rate and vorticity magnitude in the calculation of threshold cavitation pressure. This will be an important topic for futher study.

\section{ACKNOWLEDGMENTS}

This research has been done under the research project QG.21.32 "A Study on Numerical Simulation of the Vortex Ring Cavitation in Water with Consideration of Thermodynamic Effect and Concentration Air" of Vietnam Nation University, Hanoi.

\section{REFERENCES}

Allmaras, S. R., F. T. Johnson and P. R. Spalart (2012). Modification and Clarifications for the Implementation of the Spalart-Allmaras Turbulence Model. ICCFD7-1902, $7^{\text {th }}$ International Conference on Computational Fluid Dynamics.

Anh, D. L., J. Okajima and Y. Iga (2019a). Modification of Energy Equation for Homogeneous Cavitation Simulation with Thermodynamic Effect. Journal of Fluids Engineering, Transaction of the ASME, 141, 081102-1-12.

Anh, D. L., J. Okajima and Y. Iga (2019b). Numerical Simulation Study of Cavitation in Liquefied Hydrogen. Cryogenics 101, 29-35.
Anh, D. L., P. T. Hoang and T. T. Hung (2021a). Assessment of a Homogeneous Model for Simulating a Cavitating Flow in Water under a Wide Range of Temperatures. Journal of Fluids Engineering, Transaction of the ASME 143(10).

Anh, D. L. (2021b). Study of Thermodynamic Effect on the Mechanism of Flashing Flow under Pressurized Hot Water by a Homogeneous Model. Journal of Fluids Engineering, Transaction of the ASME 141(1), 011206.

Arndt, R. E. A. (2002). Cavitation in Vortical Flows. Annual Review of Fluid Mechanics 34, 143-75.

Asnaghi, A., A. Feymark and R. E. Bensow (2017). Improvement of cavitation mass transfer modeling based on local flow properties. International Journal of Multiphase Flow 93, 142-157.

Bachmann, C., V. Kini, S. Deutsch, A. A. Fontaine and J. M. Tarbell (2002). Mechanism of cavitation and the formation of stable bubbles on the Bjork-shiley monotrut prosthetic heart valve. Journal of Heart Valve Disease 11, 10513.

Bernard, P. S., J. M. Thomas and R. A. Handler (2006). Vortex dynamics and the production of Reynolds stress. Journal of Fluid Mechanics $253,385-419$.

Brandao, F., M. Bhatt and K. Mahesh (2020). Numerical study of cavitation regimes in flow over a circular cylinder. Journal of Fluid Mechanics 885, A19.

Brennen, C. E. (1974). Cavitation and Bubble Dynamics. Concepts ETI Inc. and Oxford Science Publications.

Chebli, R., B. Audebert, G. Zhang and O. CoutierDelgosha (2021). Influence of the turbulence modeling on the simulation of unsteady cavitating flows. Computers and Fluids 221, 104898 .

Coutier-Delgosha, O., R. Fortes-Patella and J. L. Reboud (2003). Evaluation of Turbulence Model Influence on the Numerical Simulation of Unsteady Cavitation. Journal of Fluids Engineering, Transaction of the ASME 125(1), $38-45$

Ducoin, A., B. Huang and Y. Yin Lu (2012). Numerical Modeling of Unsteady Cavitating Flow around a Stationary Hydrofoil. Int. J. Rotating Machinery, ID 215678.

Garam, K., C. Cheong and H. Seol (2020). Numerical Investigation of Tip-Vortex Cavitation Noise of an Elliptic Wing Using Coupled Eulerian-Lagrangian Approaches. Applied Sciences 10, 5897.

Ghahramani, E., M. H. Arabnejad and R. E. Bensow (2019). A comparative study between numerical methods in simulation of cavitating 
A. D. Le and H. T. Tran / JAFM, Vol. 15, No. 2, pp. 551-561, 2022.

bubbles. International Journal of Multiphase Flow 111, 339-359.

Ghahramani, E., H. Strom and R. E. Bensow(2021). Numerical simulation and analysis of multiscale cavitating flows. Journal of Fluid Mechanics 992.

Gnanaskandan, A. and K. Mahesh (2016). Numerical investigation of near-wake characteristics of cavitating flow over a circular cylinder. Journal of Fluid Mechanics 790, 453-491.

Iga, Y., M. Nohmi, A. Goto, B. R. Shin and T. Ikohagi (2003). Numerical Study of Sheet Cavitation Breakoff Phenomenon on a Cascade Hydrofoil. Journal of Fluids Engineering, Transaction of the ASME 125, 643-651.

Kashiwada, S. and Y. Iga (2015). Consideration of a Phase Change Model based on Apparent Phase Equilibrium. Journal of Physics: Conference Series 656, 012129

Li, C.-P., S.-F. Chen and C.-W. Lo (2012). Role of vortices in cavitation formation in the flow at the closure of a bileaflet mitral mechanical heart valve. Journal of Artificial Organs 15, 57-64.

Martynov, S. (2005). Numerical simulation of the cavitation process in Diesel fuel injector. $\mathrm{Ph} . \mathrm{D}$. thesis, The University of Brighton.

Menter, F. R. (1992). Improved Two-Equation komega Turbulence Models for Aerodynamic Flows. NASA TM 103975, Octorber.

Ochiai, N. (2011). Study of Numerical Prediction of Cavitation Erosion Based on Bubble Collapse Intensity. A dissertation submitted for the degree of Doctor of Phylosophy (Engineering), Department of mechanical Systems and Design, Tohoku University.

Pelz, P. F., T. Keil and T. F. GroB (2017). The transition from sheet to cloud cavitation. Journal of Fluid Mechanics 817, 439-454.

Rambod, E., M. Beizaie, M. Shusser, S. Milo and M. Gharib (1999). A physical model describing the mechanism for formation of gas micro- bubble in patients with mitral mechanical heart valve. Annals of Biomedical Engineering 27, 774-92.

Rouse, H. and J. S. McNown (1948). Cavitation and pressure distribution head forms at zero angle of yaw. State University of Iowa, No. 420.

Saito, Y., R. Takami, I. Nakamori and T. Ikohagi (2007). Numerical analysis of unsteady behavior of cloud cavitation around a NACA0015 foil. Computational Mechanics 40, 85-96.

Schnerr, G. H. and J. Sauer (2001). Physical and numerical modeling of unsteady cavitation dynamics. In Proceeding of the Fourth $4^{\text {th }}$ International Conferences on Multiphase Flow, New Orleans, USA.

Singhal, A., M. Athavale, H. Li and Y. Jiang (2002). Mathematical Basis and Validation of the Full Cavitation Model. Journal of Fluids Engineering, Transaction of the ASME 124(3).

Som, S., S. K. Aggarwal, E. M. El-Hannouny and D. E. Longman (2010). Investigation of nozzle flow and cavitation characteristic in a diesel injector. Journal of Engineering for Gas Turbines and Power 132(4).

Wilcox, D. C. (1994). Turbulence Modeling for $C F D$. DCW Industries, Inc., La Canada, California, USA.

Yee, H. C. (1987). Upwind and Symmetric ShockCapturing Schemes. NASA Technical Memorandum 89464.

Zwart, P. J., A. G. Gerber and T. Belamri (2004). A two-phase flow model for predicting cavitation dynamics. In Proceeding of the Fifth $5^{\text {th }}$ International Conference on Multiphase Flow, Yokohama, Japan.

Zhou, H., M. Xiang, P. N. Okolo, Z. Wu, G. J. Bennett and W. Zhang (2019). An efficient calibration approach for cavitation model constants based on OpenFOAM platform. Journal of Marine Science and Technology 24, 1043-1056. 